\title{
Influência de Aditivos na Rigidez Dielétrica do Polietileno de Alta Densidade.
}

\author{
Marcelo M. Ueki e Maria Zanin
}

Resumo: Neste trabalho são apresentados resultados de ensaios de ruptura dielétrica de curta duração com rampa de tensão positiva em amostras de polietileno de alta densidade (HDPE), modificadas com diferentes níveis de aditivos (dióxido de titânio, negro de fumo, antioxidante e estabilizante à ultravioleta). Esses resultados foram analisados estatisticamente pela distribuição de Weibull. Os parâmetros desta distribuição ( $\beta$ e $\mathrm{E}_{\gamma}$ ) foram determinados pelos métodos Gráfico e de Máxima Verossimilhança. Pôde-se concluir que os aglomerados de negro de fumo são prejudiciais à rigidez dielétrica e que o parâmetro de forma $\beta$ desta distribuição pode ser empregado para avaliar as condições de mistura dos componentes na formulação, as quais afetam a rigidez dielétrica. Por meio de microscopia ótica pôde-se verificar que o parâmetro $\beta$, quando determinado pelo método Gráfico, representa melhor a dispersão dos referidos aditivos na matriz polimérica.

Palavras-chave: Rigidez dielétrica, polietileno de alta densidade, negro de fumo, dióxido de titânio, Weibull

\section{Introdução}

O setor de distribuição de energia elétrica brasileiro, principalmente em zonas urbanas, está presenciando uma mudança grande no conceito de sistemas de distribuição de energia. Os sistemas convencionais de cabos nus, suportados por isoladores cerâmicos em cruzetas de madeira, estão sendo gradualmente substituídos por sistemas de cabos recobertos, suportados por espaçadores poliméricos denominados de redes compactas. Esta nova concepção possibilitará uma grande economia no que diz respeito à conservação do meio ambiente (poda de árvores), diminuindo o custo desta operação, além de reduzir o espaço ocupado pelos sistemas de distribuição de energia.

Dentre os vários tipos de dielétricos poliméricos, o polietileno de alta densidade (HDPE) tem se destacado como matéria prima para produção de isoladores, espaçadores e recobrimento de cabos condutores utilizados em redes de distribuição de energia elétrica. Para estas aplicações, a rigidez dielétrica é uma das propriedades que deve ser considerada para verificar a suportabilidade sob elevados campos elétricos. Ela é definida como a relação entre a tensão de ruptura e a espessura do dielétrico e representa a máxima intensidade de campo elétrico que o material pode suportar por um dado período ${ }^{[1,2]}$.

A rigidez dielétrica é determinada pelo ensaio de ruptura dielétrica e é fortemente influenciada por condições experimentais como: geometrias da amostra e do eletrodo, espessura da amostra, tempo e tipo da solicitação elétrica, meio que envolve a amostra, temperatura, etc. Além das condições experimentais, a rigidez dielétrica é afetada por variáveis como: estrutura e composição química, e microestrutura (morfologia e imperfeições) da amostra ${ }^{[1-5]}$.

O uso de polímeros de alta pureza em aplicações de engenharia é tecnologicamente inviável o que leva 
ao desenvolvimento de formulações à base de aditivos que protegem os polímeros contra perdas em suas propriedades (por exemplo, mecânicas e termomecânicas) durante as etapas de processamento e/ou sob uso $^{[6]}$. Estes aditivos, no caso das aplicações em isolação elétrica podem atuar de forma prejudicial ou não sobre as propriedades elétricas.

KOLESOV $^{[4]}$, e, KITAGAWA e colaboradores ${ }^{[5]}$ utilizaram, respectivamente, aditivos como ceresina e antioxidante (atuando como agentes nucleantes), e verificaram um aumento substancial na rigidez dielétrica do polietileno. Segundo os autores, a redução do tamanho dos esferulitos, causada pela incorporação destes aditivos, proporciona um aumento da densidade da fase amorfa, resultando num menor livre caminho médio e consequentemente menor velocidade média dos portadores de carga. KITAGAWA e colaboradores ${ }^{[5]}$ adicionaram também pirina ao polietileno, composto rico em elétrons $\pi$, porém com pouca ação como agente nucleante, e verificaram um aumento acentuado na rigidez dielétrica, que atribuíram à capacidade dos elétrons $\pi$ estabilizarem elétrons livres. YOSHINO e colaboradores ${ }^{[7]}$ estudaram a rigidez dielétrica à impulso, à temperatura ambiente, do polietileno de baixa densidade dopado com iodo $\left(\mathrm{I}_{2}\right)$. Verificaram a diminuição desta, de $6 \mathrm{MV} / \mathrm{cm}$ para 2,5 MV/cm, que atribuíram ao aumento da mobilidade dos portadores na fase amorfa do material.

KHALIL e colaboradores ${ }^{[8]}$ ensaiaram polietileno de baixa densidade com $1 \%$ de dióxido de titânio, em degraus de tensão positiva (DC) e verificaram uma diminuição de $10 \%$ na rigidez dielétrica do polietileno dopado $(6,5 \mathrm{MV} / \mathrm{cm})$ em relação ao polietileno puro $(7,1 \mathrm{MV} / \mathrm{cm})$. Eles correlacionaram este fato à mudança da distribuição de carga espacial no material com $\mathrm{TiO}_{2}$, passando de homocargas balanceadas no polietileno puro para distribuição dominada por cargas negativas no polietileno com $\mathrm{TiO}_{2}$. Tal fato proporciona um aumento do campo elétrico em um dos eletrodos levando o material a romper em tensões menores. Ao ensaiar o polietileno dopado com reversão de polaridade, não verificaram mudanças significativas na rigidez dielétrica $(6,3 \mathrm{MV} / \mathrm{cm})$. Segundo os autores, pelo fato da mudança na distribuição de carga espacial ocorrer lentamente, a carga espacial se mantém "congelada" enquanto os eletrodos mudam de polaridade, proporcionando o aumento do campo no outro eletrodo, e deste modo, a mesma probabilidade de ruptura.

Um aspecto importante do dióxido de titânio (tipo rutilo) é a sua capacidade de proteção à radiação ultravioleta (UV) em poliolefinas ${ }^{[9-11]}$. No caso do polipropileno, 2,0\% de dióxido de titânio equivale à proteção oferecida por $0,2 \%$ de negro de fumo ${ }^{[10]}$, que se combinado com um estabilizante UV do tipo aminas estericamente impedidas (HALS), proporciona sinergismo bastante pronunciado na proteção à radiação ultravioleta ${ }^{[9,10]}$.

Gumin e Zakgein, citados por KU e LIEPINS ${ }^{[1]}$, verificaram que a introdução de $1 \%$ de negro de fumo em resinas (não citou o tipo de resina) diminui a rigidez dielétrica em $90 \%$, enquanto níveis superiores a $1 \%$ não propiciam quedas significativas na mesma. Segundo os autores, este comportamento é devido à grande diferença entre a condutividade e a constante dielétrica da resina e do negro de fumo, que causa distorções no campo elétrico e resulta na diminuição da rigidez dielétrica.

OKAMOTO e colaboradores ${ }^{[12]}$ estudaram o efeito do tamanho de aglomerados de negro de fumo presentes na região de interface, entre a camada semicondutora ( $\operatorname{com} 35 \%$ em peso de negro de fumo) e o isolante (polietileno reticulado sem negro de fumo), na rigidez dielétrica a degraus de tensão (AC - $50 \mathrm{~Hz}$ ). Para modificar o tamanho dos aglomerados, utilizaram diferentes aditivos (à base de glicerídeos) e constataram que existe um tamanho ótimo destes, em torno de $200 \mathrm{~nm}$, em que se observa valores elevados de rigidez dielétrica.

Mesmo que variáveis como morfologia, aditivos ou contaminantes e condições de ensaio estejam sob controle, a dispersão dos resultados de ensaio de rigidez dielétrica é inevitável, sendo necessário: a realização de réplicas, utilizar modelos estatísticos para tratar e obter o valor da rigidez dielétrica e ainda avaliar a sua significância para garantir a confiabilidade desse valor. Dos possíveis modelos estatísticos, o de Weibull é considerado o mais adequado para tratar os dados obtidos a partir dos ensaios de ruptura dielétrica $^{[2,12,13]}$.

COPPARD e colaboradores ${ }^{[14]}$ verificaram que o parâmetro de forma $\beta$ da distribuição de Weibull pode avaliar a dispersão dos resultados, a qual está relacionada com a distribuição de tamanho dos defeitos que, por sua vez afetam a rigidez dielétrica. Eles concluíram que quanto menor a dispersão dos resultados, mais estreita a distribuição de tamanho dos defeitos e maior o valor de $\beta$.

Baseado nestas considerações, este trabalho tem como objetivo avaliar a influência de aditivos (negro de fumo, dióxido de titânio, estabilizante UV e 
antioxidantes), isoladamente ou combinados, no comportamento de ruptura dielétrica do polietileno de alta densidade (HDPE), através de ensaio com rampa de tensão positiva e eletrodos esfera-plano. As medidas de rigidez dielétrica obtidas serão tratadas estatisticamente empregando a distribuição de Weibull, buscando, desta maneira, o fornecimento de subsídios para a utilização segura destes aditivos quando presentes em sistemas de isolação elétrica.

\section{Materiais e Métodos}

\section{Materiais}

O HDPE utilizado, fabricado e fornecido pela POLIALDEN, possui índice de fluidez (MFI) 0,9g/ 10min. (condição E da norma ASTM D1238). Para este estudo foram utilizados dois aditivos de estabilização: IRGANOX B215 (estabilizante térmico) e TINUVIN 111 (estabilizante à radiação ultravioleta), ambos fornecidos pela CIBA-GEIGY, e dois pigmentos: negro de fumo e dióxido de titânio, simbolizados por $\mathrm{N} 220$ e $\mathrm{TiO}_{2}$, respectivamente. Tanto os estabilizantes quanto os pigmentos foram incorporados ao polímero puro na forma de concentrado ("masterbatch"). Os pigmentos foram fornecidos pela CROMEX, já na forma de concentrados na proporção de $30 \%$ em peso. O veículo destes concentrados foi um HDPE com MFI de 1,1g/10min. O masterbatch do antioxidante, simbolizado por $\mathrm{AO}$ e do estabilizante UV (UV), foram desenvolvidos em nosso laboratório através de um reômetro de torque HAAKE HBI System 90, tendo como veículo um HDPE com MFI = 1,1 g/10min. Após a incorporação dos aditivos ao veículo, os "masterbatches", ainda no estado fundido, foram conformados na forma de chapas que posteriormente foram picotadas.

\section{Preparação das Amostras}

Os aditivos na forma de "masterbatches" foram incorporados ao polímero virgem por extrusão, em extrusora de rosca única Gerst. O material extrudado passou por secagem durante 72 horas à temperatura ambiente e foi posteriormente granulado numa picotadora CONAIR/JETRO modelo 304. A partir do granulado, filmes com espessuras variadas foram obtidos por compressão à quente, depositando os gra-
Tabela 1. Concentração dos aditivos das formulações (níveis em \% de peso).

\begin{tabular}{ccccc}
\hline Form. & $\mathrm{N} 220$ & $\mathrm{TiO}_{2}$ & $\mathrm{AO}$ & $\mathrm{UV}$ \\
\hline $\mathrm{A} 00$ & 2,0 & 0,5 & 0,05 & - \\
$\mathrm{A} 01$ & 2,0 & 0,5 & 0,05 & 0,20 \\
$\mathrm{~A} 10$ & 2,0 & 0,5 & 0,20 & - \\
$\mathrm{A} 11$ & 2,0 & 0,5 & 0,20 & 0,20 \\
B00 & 0,5 & 2,0 & 0,05 & - \\
B01 & 0,5 & 2,0 & 0,05 & 0,20 \\
B10 & 0,5 & 2,0 & 0,20 & - \\
B11 & 0,5 & 2,0 & 0,20 & 0,20 \\
\hline
\end{tabular}

nulados entre dois filmes de poliéster colocados entre duas placas metálicas de aço inox polidas. Este conjunto, colocado entre as placas de uma prensa, pré-aquecidas a $180^{\circ} \mathrm{C}$ foi submetido a uma pressão em torno de $10 \mathrm{kgf} / \mathrm{cm}^{2}$, suficiente para produzir filmes com espessuras entre 40 e $100 \mu \mathrm{m}$.

Para o planejamento experimental (níveis dos aditivos), optou-se por desenvolver 2 blocos, com 4 formulações em cada bloco, e o fatorial completo em dois níveis dentro de cada bloco. As formulações seguem a designação "AXY" e "BXY" onde: "A" e "B" indicam os blocos ("A" $=2,0 \%$ de $\mathrm{N} 220$ e $0,5 \%$ de $\mathrm{TiO}_{2}$ e "B" $=0,5 \%$ de $\mathrm{N} 220$ e $2,0 \%$ de $\mathrm{TiO}_{2}$ ), "X" o nível de antioxidante (zero para o nível inferior e 1 para o superior) e "Y" o nível de estabilizante UV, seguindo a mesma designação de "X". A Tabela 1 apresenta as concentrações de aditivos utilizadas para as formulações estudadas.

\section{Ensaio Elétrico}

Os ensaios de rigidez dielétrica foram realizados em um sistema desenvolvido no Laboratório de Propriedades Elétricas de polímeros do DEMa/UFSCar. O sistema é automatizado por um microcomputador 486DX2, que controla uma fonte de tensão BERTAN modelo 225, cuja função é aplicar a tensão nos eletrodos. Este sistema permite o controle via computador da taxa de aplicação da tensão, interrupção automática no instante da ruptura e seleção da forma de onda do sinal a ser aplicado à amostra. Para este trabalho, o tipo de solicitação elétrica foi uma rampa de tensão positiva, com taxa de subida de $500 \mathrm{~V} / \mathrm{seg}$., aplicada entre eletrodos esfera-plano imersos em óleo de silicone à temperatura entre 20 e $22^{\circ} \mathrm{C}^{[15]}$. 
A espessura da amostra foi determinada após o ensaio de rigidez nas proximidades do ponto de ruptura através do medidor PERMASCOPE MPO, via indução magnética. Com a intenção de controlar a variável espessura na análise dos resultados foram considerados os vinte primeiros resultados, cujos valores se encontravam entre 50 e $70 \mu \mathrm{m}$.

\section{Determinação do Grau de Cristalinidade ${ }^{[16,17]}$}

O grau de cristalinidade foi determinado para as diferentes formulações utilizando dados de difração de raios-X, obtidos de filmes com espessura em torno de $100 \mu \mathrm{m}$ através de um difratômetro PHILLIPS modelo X'PERT MPD. O método utilizado neste trabalho baseia-se na possibilidade de separar as contribuições das fases amorfa e cristalina no espalhamento de raios-X. Assim o grau de cristalinidade foi obtido pela razão entre a soma das áreas dos picos de difração relativos à fase cristalina e a área total, definida arbitrariamente num intervalo de ângulo de espalhamento.

Dois picos intensos, relativos à fase cristalina a $21,6^{\circ}$ (110) e a $24,0^{\circ}(200)$, e uma banda amorfa centrada em aproximadamente $19^{\circ}$ foram considerados. A região do difratograma analisada esteve entre 15 e $30^{\circ}$.

$\mathrm{O}$ valor do grau de cristalinidade utilizado neste estudo corresponde à média de três resultados calculados pela equação 1 .

$$
\mathrm{W}_{\mathrm{c}, \mathrm{x}}=\frac{\mathrm{S}_{(110)}+\mathrm{S}_{(200)}}{\mathrm{S}_{(110)}+\mathrm{S}_{(200)}+\mathrm{S}_{\text {amorfa }}}
$$

onde: $S_{(110)}$ e $S_{(200)}$ são as áreas relativas dos picos correspondentes aos planos de difração (110) e (200), respectivamente, e $S_{\text {amorfa }}$ está relacionada à área da banda amorfa.

\section{Distribuição de Weibull}

O modelo de Weibull de dois parâmetros $\left(\beta\right.$ e $\left.\mathrm{E}_{\gamma}\right)$ foi utilizado para avaliar os resultados dos ensaios de rigidez dielétrica. A equação 2 apresenta a função distribuição acumulada de Weibull adotada:

$$
P_{f}=1-\exp \left[-\left(\frac{E_{\text {rup }}}{E_{\gamma}}\right)^{\beta}\right]
$$

onde: $P_{f}$ é a probabilidade acumulada de falha, $E_{r u p}$ a rigidez dielétrica $(\mathrm{MV} / \mathrm{cm}), E_{\gamma}$ a rigidez dielétrica para
$P_{f}=63,2 \%$, denominada de parâmetro de escala, e $\beta$ o parâmetro de forma. Comumente, o valor $E_{\gamma}$ é utilizado para comparar valores de rigidez dielétrica determinados nos ensaios de ruptura elétrica ${ }^{[1,2,12-14]}$.

Os parâmetros desta distribuição foram determinados pelo Método Gráfico e pelo Método da Máxima Verossimilhança.

\section{Método Gráfico}

Os coeficientes $\beta$ e $E_{\gamma}$ foram determinados a partir da regressão linear da equação 2 quando linearizada para:

$$
\log \left(-\operatorname{Ln}\left(1-P_{f}\right)\right)=\beta \cdot \log \left(E_{\text {rup }}\right)-\beta \cdot \log \left(E_{\gamma}\right)
$$

Conforme recomendação da norma IEC 56, empregou-se a função distribuição apresentada na equação 4.

$$
\mathrm{P}_{\mathrm{f}}=(\mathrm{i}-0,5) /(\mathrm{n}+0,25)
$$

onde: $i$ é o $i$-ésimo resultado quando os valores de $E_{\text {rup }}$ são colocados em ordem crescente e $n$ o número de pontos (para este trabalho, $n=20$ ).

\section{Método da Máxima Verossimilhança}

O método da Máxima Verossimilhança é considerado atualmente o mais importante estimador de parâmetros $^{[18]}$. Para estimar $\beta$ e $E_{\gamma}$, deve-se resolver a equação 5:

$$
\frac{\mathrm{n}}{\hat{\beta}}-\mathrm{n} \frac{\sum_{\mathrm{i}=1}^{\mathrm{n}} \mathrm{E}_{\text {rup }, \mathrm{i}} \cdot \ln \mathrm{E}_{\text {rup }, \mathrm{i}}}{\sum_{\mathrm{i}=1}^{\mathrm{n}} \mathrm{E}_{\text {rup }, \mathrm{i}}}+\sum_{\mathrm{i}=1}^{\mathrm{n}} \ln \mathrm{E}_{\text {rup }, \mathrm{i}}=0
$$

onde: $E_{\text {rup }, i}$ é o $i$-ésimo valor de rigidez dielétrica, $\hat{\beta}$ o valor estimado de $\beta$ e $n$ o número de amostras.

Determinado o valor de $\hat{\beta}$, o valor estimado $E_{\gamma}$ é obtido pela equação (6):

$$
\hat{\mathrm{E}}_{\gamma}=\left[\sum_{\mathrm{i}=1}^{\mathrm{n}} \frac{\left(\mathrm{E}_{\text {rup }, \mathrm{i}}\right) \cdot \hat{\beta}}{\mathrm{n}}\right]^{1 / \hat{\beta}}
$$

Para resolver as equações. (5) e (6), foi utilizado o método interativo presente no programa EXCEL 7.0. O intervalo de confiança para $E_{\gamma}$ à $95 \%$ também foi determinado. 


\section{Resultados e Discussões}

\section{Análise dos Resultados de Rigidez Dielétrica}

\section{Parâmetro de Escala $\left(\mathrm{E}_{\gamma}\right.$.}

A Figura 1 apresenta os resultados de rigidez dielétrica $\left(E_{\text {rup. }}\right)$ linearizados pela equação 4 . Inicialmente nota-se a formação de três conjuntos distintos de famílias de pontos: um correspondente às formulações "AXY" (2,0\% de $\left.\mathrm{N} 220,0,5 \% \mathrm{de} \mathrm{TiO}_{2}\right)$, outro às formulações "BXY" $\left(0,5 \%\right.$ de $\mathrm{N} 220,2,0 \%$ de $\left.\mathrm{TiO}_{2}\right)$ e o terceiro correspondendo ao HDPE puro (BR). Ainda pode-se observar que existe um certo grau de dispersão nos resultados de rigidez dielétrica em todas as formulações estudadas, principalmente nas A00 e B11.

Os três conjuntos de pontos podem ser melhor visualizados na Figura 2 onde tem-se os dados de $E_{\gamma}$ para cada formulação com seus respectivos intervalos de confiança à $95 \%$, obtidos pelo método de Máxima Verossimilhança. Analisando os valores de $E_{\gamma} \mathrm{e}$ seus respectivos intervalos de confiança apresentados na Figura 2, fica comprovada estatisticamente a formação dos três conjuntos pois nenhum resultado de $E_{\gamma}$, do bloco "A" superpõe aos do bloco "B" e viceversa. $\mathrm{O}$ mesmo raciocínio é válido para $\mathrm{BR}$.

Com a incorporação de $0,5 \%$ de N220 e 2,0\% de $\mathrm{TiO}_{2}$, verificou-se em ambos os métodos uma diminuição da rigidez dielétrica em torno de $35 \%$ quando comparada à do polietileno puro (aprox. 5,9 MV/cm). Para 2,0\% de $\mathrm{N} 220$ e $0,5 \%$ de $\mathrm{TiO}_{2}$, uma diminuição de até $57 \%$ foi verificada.

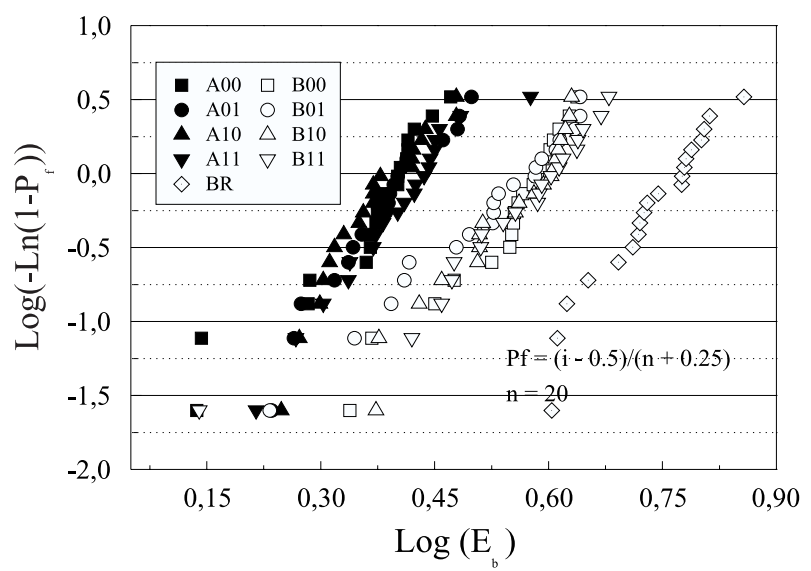

Figura 1. Linearização do modelo de Weibull para rigidez dielétrica de HDPE com aditivos. $E_{\text {rup. }}=$ rigidez dielétrica $(\mathrm{MV} / \mathrm{cm})$ e $P_{f}$ a função probabilidade acumulada de falha.

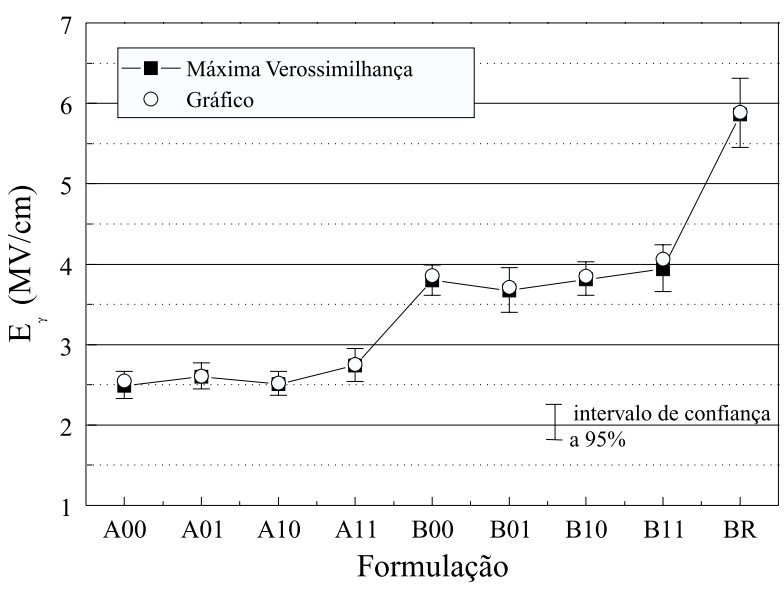

Figura 2. Valores de $E_{\gamma}$ obtidos pelo método de Máxima Verossimilhança e pelo método Gráfico ${ }^{[19]}$.

Estes resultados sugerem que o componente mais significativo na rigidez dielétrica é o teor de negro de fumo, praticamente não se observando alterações significativas na rigidez dielétrica com a variação no nível dos outros componentes (antioxidante e estabilizante UV) incorporados ao HDPE. Com a finalidade de verificar se o dióxido de titânio também pouco afeta a rigidez dielétrica do HDPE, duas formulações foram desenvolvidas especialmente: uma contendo $2,5 \%$ de $\mathrm{TiO}_{2}$ denominada de $\mathrm{T} 11$, e outra com $2,5 \%$ de negro de fumo denominada de N11, ambas com $0,20 \%$ de antioxidante e $0,20 \%$ de estabilizante UV.

A Figura 3 mostra os valores de rigidez dielétrica obtidos destas duas novas formulações. Para efeito de comparação, os resultados das formulações de mesmo nível de antioxidante e estabilizante UV (A11 e B11) e do HDPE puro (BR) também são apresentados.

Analisando a Figura 3, observa-se que o menor valor de rigidez dielétrica determinado experimentalmente nas amostras B11 se encontra entre os menores valores de A11 e N11. Esta observação é importante pois a ruptura dielétrica que ocorre pelo volume do dielétrico sempre se manifesta no ponto mais fraco. Segundo Okamoto $^{[12,14]}$, provavelmente ela não ocorra no valor de $E_{\gamma}\left(P_{f}=63,2 \%\right)$ mas em valores bem menores, por exemplo, em $E_{r u p}$. para $P_{f}=1 \%$. Deste modo, afirmar que o isolante com maior valor de $E_{\gamma}$ é o recomendado não é conveniente já que na prática este poderá falhar em níveis de campo elétrico inferiores a este.

A Tabela 2 apresenta os valores de $E_{\gamma}$, obtidos pelo método de Máxima Verossimilhança e pelo Gráfico, para as formulações especiais com $2,5 \% \mathrm{de} \mathrm{TiO}_{2}$ (T11) e com 2,5\% de N220 (N11) e para as A11, B11 e BR. 


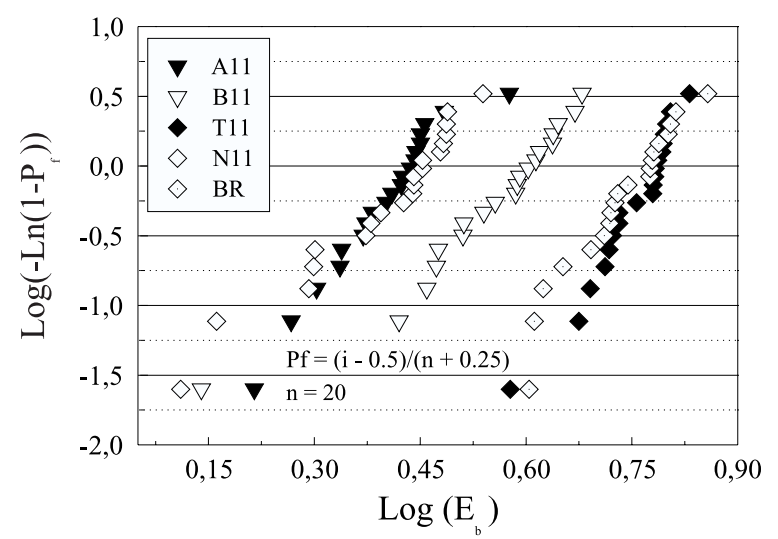

Figura 3. Linearização do modelo de Weibull. Comparação entre diferentes níveis de pigmentos $\left(\mathrm{TiO}_{2}\right.$ e $\left.\mathrm{N} 220\right)$.

Comparando os valores de $E_{\gamma}$ (Tabela 2) das formulações com diferentes concentrações de N220 (A11 e N11) observa-se que estes são similares, mostrando que o aumento de $2,0 \%$ para $2,5 \%$ na concentração de negro de fumo não afeta significativamente a rigidez dielétrica. Porém ambas as formulações apresentaram $E_{\gamma}$ inferiores a B11, o que confirma novamente a maior influência do negro de fumo nesta propriedade.

Com relação à formulação T11, o valor de $E_{\gamma}$ apresenta-se ligeiramente superior ao do BR (cerca de $4 \%$ ), porém sem significância já que ambos $E_{\gamma}$ 's estão simultaneamente dentro do intervalo de confiança de $95 \%$ (Tabela 2). Este pequeno aumento, a rigor, contraria os resultados observados por KHALIL e colaboradores ${ }^{[8]}$ que observaram uma significativa diminuição da rigidez dielétrica na presença de dióxido de titânio. Esta pequena discordância de resultados pode ser atribuída a dois fatores: sistema de eletrodos e porta-amostras diferentes do utilizado neste trabalho e o polietileno por eles estudado ser de baixa densidade (LDPE).

Os dados da Tabela 2 foram plotados na Figura 4 para melhor visualização destes.

Tabela 2. - Valores de $E_{\gamma}(\mathrm{MV} / \mathrm{cm})$ determinados pelos métodos de Máxima Verossimilhança e Gráfico

\begin{tabular}{|c|c|c|c|c|}
\hline \multirow[t]{3}{*}{ Formul. } & \multicolumn{3}{|c|}{ Máxima Verossimilhança } & $\begin{array}{l}\text { Método } \\
\text { Gráfico }\end{array}$ \\
\hline & \multirow[b]{2}{*}{$\mathrm{E}_{\gamma}$} & \multicolumn{2}{|c|}{$95 \%$ confiança } & \\
\hline & & $\mathrm{E}_{\gamma \text { sup }}$ & 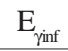 & $\mathrm{E}_{\gamma}$ \\
\hline T11 & 5,99 & 6,30 & 5,69 & 6,04 \\
\hline N11 & 2,78 & 3,05 & 2,53 & 2,83 \\
\hline A11 & 2,74 & 2,95 & 2,54 & 2,75 \\
\hline B11 & 3,94 & 4,24 & 3,66 & 4,06 \\
\hline BR & 5,86 & 6,30 & 5,45 & 5,89 \\
\hline
\end{tabular}

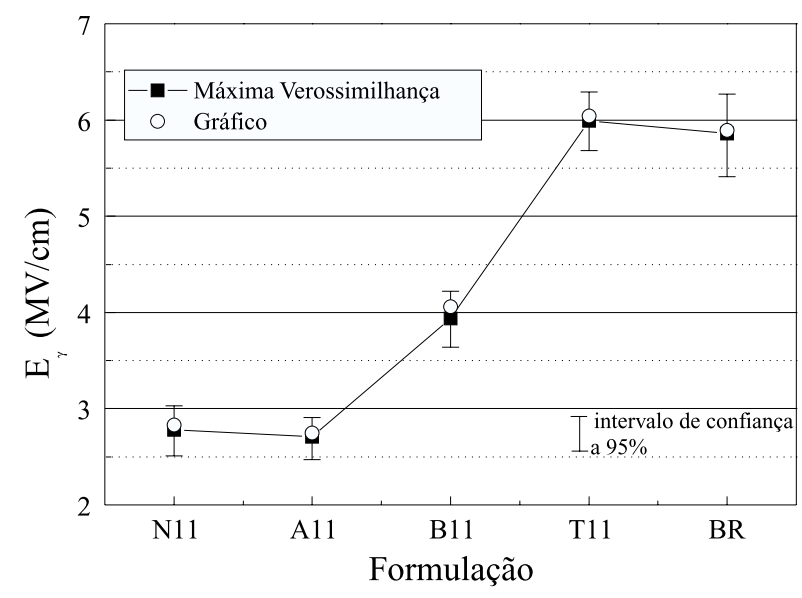

Figura 4. Valores de $E_{\gamma}$ obtidos pelo método de Máxima Verossimilhança e pelo método Gráfico para formulações com diferentes níveis de pigmentos $\left(\mathrm{TiO}_{2}\right.$ e negro de fumo). Intervalo de confiança à $95 \%$ para $E_{\gamma}$ obtido pelo método de Máxima Verossimilhança.

\section{Parâmetro de Forma $\beta$}

A Tabela 3 apresenta os valores de $\beta$, determinados pelo método Gráfico e pelo da Máxima Verossimilhança, já corrigidos para pequeno número de réplicas e de $\Delta E$ (diferença entre $\log \left(E_{\gamma}\right)$ e $\log \left(E_{\text {min }}\right)$ ), que representa o espalhamento dos resultados determinados experimentalmente para cada formulação. $E_{\min }$ é o menor valor medido de rigidez dielétrica. $\mathrm{Ob}-$ servando a Tabela 3, nota-se diferenças superiores a $20 \%$ entre os parâmetros $\beta$ calculados pelos diferentes métodos. Este fato mostra a necessidade de se adotar critérios na escolha do método utilizado para estimar o valor de $\beta$, conforme já observado por Ross ${ }^{[13]}$.

O método de Máxima Verossimilhança embora bastante preciso para estimar os parâmetros da distribuição de Weibull, é pouco sensível à dispersão

Tabela 3. Parâmetro $\beta$ determinado pelo método da Máxima Verossimilhança e Gráfico

\begin{tabular}{c|cc|cc}
\hline & \multicolumn{2}{|c|}{$\begin{array}{c}\text { Máxima } \\
\text { Verossimilhança }\end{array}$} & \multicolumn{2}{c}{ Método Gráfico } \\
\hline & $\beta$ & $\Delta \mathrm{E}$ & $\beta$ & $\Delta \mathrm{E}$ \\
\hline A00 & 7,3 & 0,259 & 5,7 & 0,270 \\
A01 & 6,5 & 0,179 & 7,3 & 0,181 \\
A10 & 6,8 & 0,152 & 7,8 & 0,153 \\
A11 & 5,3 & 0,223 & 6,5 & 0,224 \\
B00 & 7,9 & 0,242 & 6,4 & 0,248 \\
B01 & 5,2 & 0,332 & 4,8 & 0,336 \\
B10 & 7,1 & 0,208 & 6,3 & 0,213 \\
B11 & 5,4 & 0,456 & 4,2 & 0,469 \\
BR & 6,9 & 0,164 & 7,4 & 0,166 \\
\hline
\end{tabular}




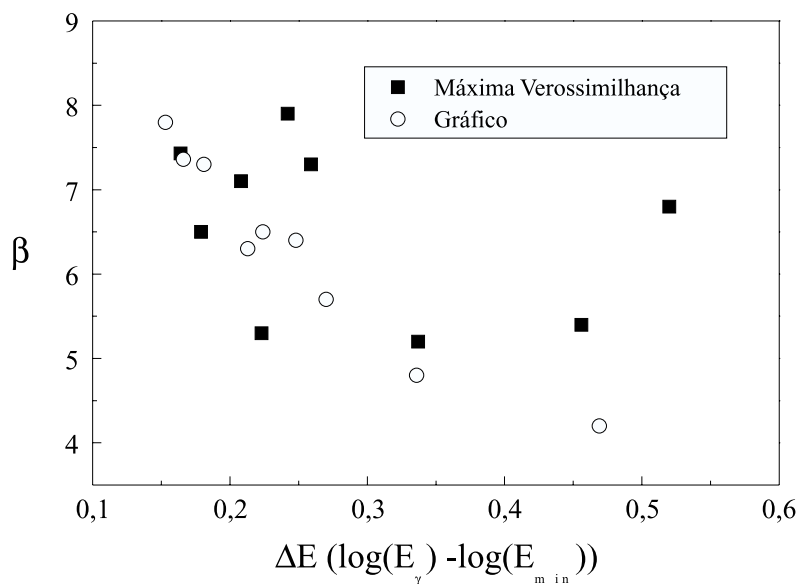

Figura 5. Relação de $\beta$ com a diferença entre $\log \left(E_{\gamma}\right)$ e $\log \left(E_{\text {min }}\right)$ para formulações apresentadas na Tabela 3.

dos dados ${ }^{[13]}$. A Figura 5 ilustra os valores presentes na Tabela 3, mostrando uma tendência em diminuir os valores de $\beta$, determinados pelo método Gráfico, com o aumento de $\Delta E$; enquanto que para $\beta$ 's calculados pelo método de Máxima Verossimilhança é verificado um comportamento aleatório.

Por esta análise, ao considerar duas formulações com mesmo $E_{\gamma}$ e diferentes $\beta$ 's, é possível concluir que a que possui maior valor de $\beta$, determinado pelo método Gráfico, apresenta maior confiabilidade (qualidade), durante a sua utilização, em relação ao de menor $\beta^{[12,14]}$.

COPPARD e colaboradores ${ }^{[14]}$, verificaram que $\beta$ está relacionado com a distribuição de tamanho das impurezas presentes no dielétrico. Para o trabalho apresentado aqui, significa que $\beta$ está relacionado com a distribuição/dispersão do(s) aditivo(s) que influencia $(\mathrm{m})$ na rigidez dielétrica mais especificamente com a do negro de fumo, em termos de distribuição de tamanho de aglomerados. Esta afirmação é validada quando se observa, através de um microscópio ótico, o local onde ocorreu a ruptura. As Figuras 6a, 6b e 6c ilustram algumas das amostras ensaiadas. Os pontos pretos são os aglomerados de negro de fumo e a seta branca indica a perfuração provocada pela descarga elétrica mostrando que os aglomerados são os pontos mais susceptíveis à ruptura.

Portanto, $\beta$ é influenciado pelas variáveis que afetam as condições de incorporação (mistura) dos aditivos, como por exemplo: qualidade do "masterbatch" ${ }^{[20]}$ e condições de processamento ${ }^{[20,22]}$.

Além de afetar a rigidez dielétrica, o controle da dispersão dos aditivos, como negro de fumo, é importante para tornar efetivo a proteção de poliolefinas à radiação $\mathrm{UV}^{[20-22]}$.

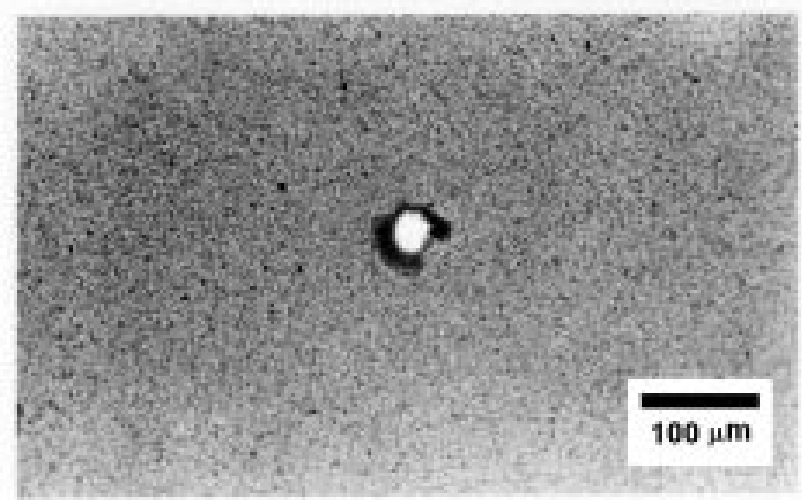

Figura 6a. Formulação N11. Tensão de ruptura $=2817$ V. Rigidez Dielétrica $=1,76 \mathrm{MV} / \mathrm{cm}$. Aumento $=250 \mathrm{X}$.

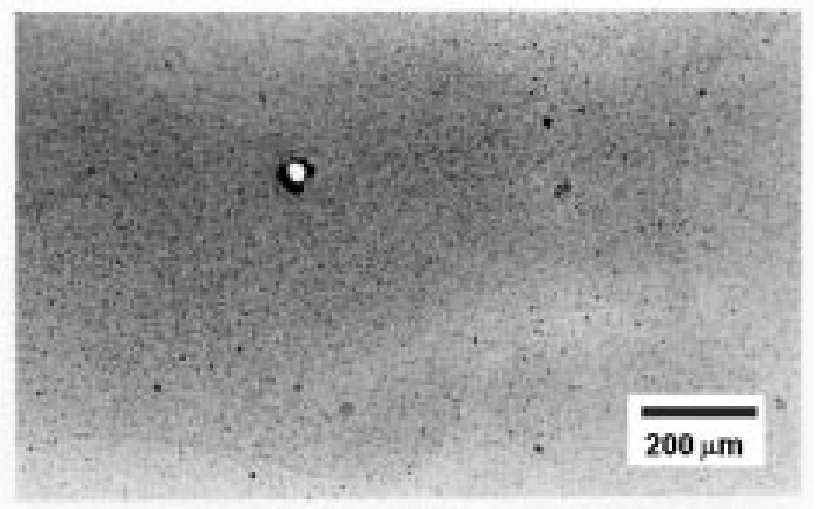

Figura 6b. Formulação N11. Tensão de ruptura $=2817$ V. Rigidez Dielétrica $=1,76 \mathrm{MV} / \mathrm{cm}$. Aumento $=125 \mathrm{X}$.

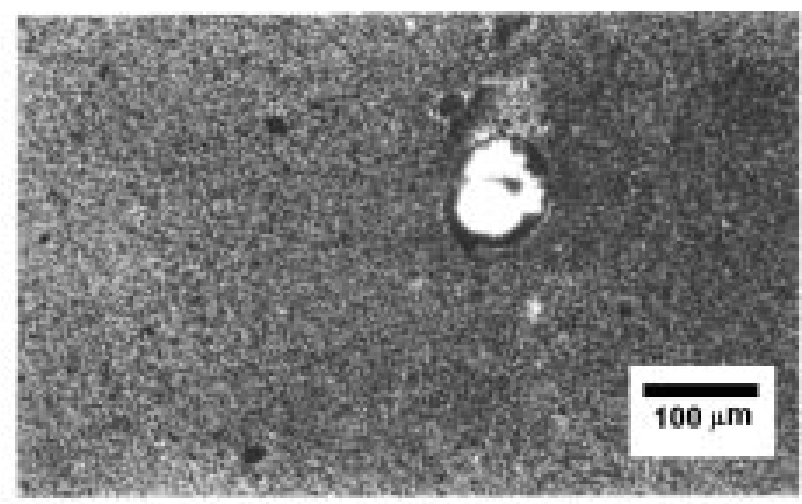

Figura 6c. Formulação A11. Tensão de ruptura $=4478$ V. Rigidez Dielétrica $=1,36 \mathrm{MV} / \mathrm{cm}$. Aumento $=250 \mathrm{X}$.

\section{Grau de Cristalinidade}

Outro fator que pode influenciar os resultados da rigidez dielétrica é o grau de cristalinidade ${ }^{[1,3,4]}$. A Figura 7 mostra as porcentagens de cristalinidade, $W_{c, x}$, obtidas a partir de dados de difração de raios-X para as diferentes formulações. Os valores de $W_{c, x}$ encontram-se entre 66 e $68 \%$ para todas as formulações, indicando que a variação dos resultados de rigidez dielétrica nas diferentes formulações não deve 


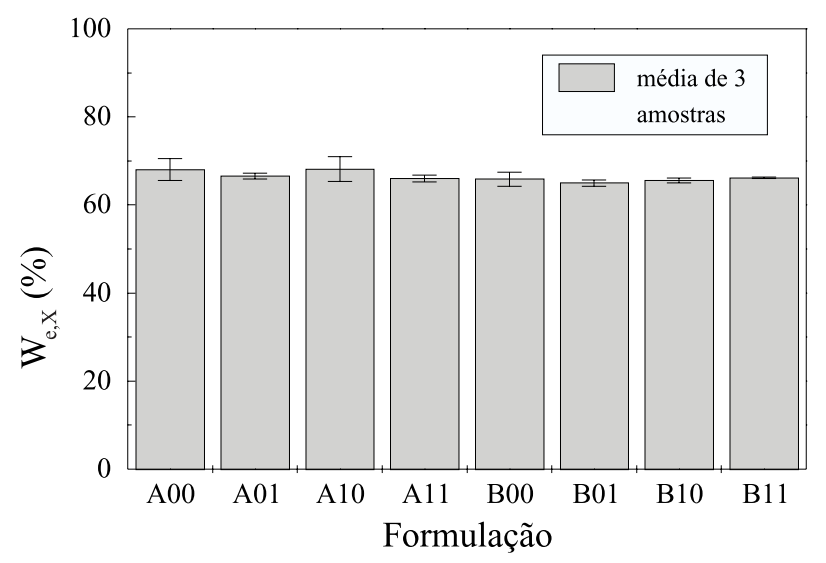

Figura 7. Grau de cristalinidade das formulações estudadas.

ter sido causada por esta variável e sim pela mudança no teor do negro de fumo.

No caso da diferença de cristalinidade para três amostras de mesma formulação verificou-se que a maior variação sobre a média não foi superior o $5 \%$, indicando que este fator não é tão significativo quanto a distribuição de tamanho de aglomerados de negro de fumo sobre a dispersão dos resultados de rigidez dielétrica.

Por esta análise, do ponto de vista da rigidez dielétrica e da confiabilidade do dielétrico durante o seu uso, a formulação contendo somente dióxido de titânio é a mais indicada por apresentar simultaneamente, $\beta$ ( 8,9 pelo mét. Gráfico e 10,0 pelo mét. Máx. Verossimilhança) e $E_{\gamma}(5,99$ e $6,04 \mathrm{MV} / \mathrm{cm}$ da Tabela 2) superiores aos obtidos nas outras formulações.

\section{Conclusões}

A partir deste estudo pode-se concluir que o componente negro de fumo é o fator crítico para a rigidez dielétrica do polietileno de alta densidade. Este fato foi comprovado por microscopia ótica onde observou-se que os canais de ruptura surgem preferencialmente nos aglomerados de negro de fumo. O dióxido de titânio, nas concentrações utilizadas, não causa mudanças significativas na rigidez dielétrica, sendo um bom candidato para substituir o negro de fumo em materiais dielétricos que atualmente o utilizam como pigmento e protetor à radiação UV.

É possível concluir também que o valor do parâmetro de forma $\beta$ da distribuição de Weibull, quando determinado pelo método Gráfico, pode ser utilizado para avaliar as condições de distribuição do tamanho dos defeitos (no caso, aglomerados de ne- gro de fumo) que prejudicam a rigidez dielétrica do isolante, estando diretamente relacionado à dispersão deste na resina polimérica em questão. Maior valor de $\beta$, menor espalhamento dos resultados de rigidez dielétrica, melhor a dispersão do negro de fumo e consequentemente maior a confiabilidade da característica dielétrica do material.

\section{Agradecimentos}

Os autores agradecem:

Ao Eng. Jorge N. dos Santos (bolsista DTI/CNPq no DEMa/UFSCar) pelo desenvolvimento do sistema de ensaio de rigidez dielétrica; ao Prof. Dr. José A. M. Agnelli (DEMa/UFSCar); ao corpo técnico do CNMT/LAC/COPEL pelos ensaios de raios-X; à POLIALDEN, CROMEX e CIBA-GEIGY pelo fornecimento dos materiais e à FAPESP pelo financiamento do projeto 94/2640-2.

\section{Referências Bibliográficas}

1. Ku, C. C. e Liepins, R. Dielectric Breakdown of Polymers. In: Electrical Properties of Polymers - Chemical Principles, Munich: Hanser Publishers, p. 102-199, (1987).

2. Bartnikas, R. ed.; "Engineering Dielectrics, vol. IIB, Electrical Properties of Solid Insulating Materials: Measurement Techniques", Philadelphia: ASTM Special Technical Publication No. 926, 589 p, (1987).

3. Ieda, M.; IEEE Trans. Electr. Insul., EI - 15, n. 3, p. 206-224 (1980).

4. Kolesov, S. N.; IEEE Trans. Electr. Insul., EI - 15, n. 5, p. 382-388 (1980).

5. Kitagawa, K. Sawa, G. e Ieda, M., Elect. Eng. Japan, 109A, n. 2, p. 42-51 (1989).

6. Gächter, R. e Müller, H.; "Plastic Additives Handbook", 3e ed. , Munich: Hanser Publishers, $970 \mathrm{p},(1990)$.

7. Yoshino, K., Harada, S., Kyokane, J., Iwakawa, S. e Inuishi, Y., J. Appl. Phys., 51, n. 5, p. 27142717 (1980).

8. Khalil, M. S., Henk, P. O. e Henriksen, M., Conference Record of the 1990 IEEE 
International Symposium on Electrical Insulation, p. 268-271, Canada, June (1990).

9. Day, R. E., Polym. Degrad. and Stabil., 29, p. 7392 (1990).

10. RyŠavý, D. e Tkadlecková, H., Polym. Degrad. and Stabil., 37, p. 19-23 (1992).

11. Reeve, T. B., Plastics Eng., august, p. 31-37 (1977).

12. Okamoto, T., Ishida, M. e Hozumi, N., IEEE Trans. Electr. Insul., 23, n. 3, p. 335-344 (1988).

13. Ross, R.; IEEE Trans. Electr. Insul., 1, n. 2, p. 247-253 (1994).

14. Coppard, R. W. et al.; J. Phys. D: Appl. Phys., n. 23, p. 1554-1561 (1990).

15. Ueki, M. M. "A influência de aditivos na rigidez dielétrica do polietileno de alta densidade.", Dissertação de mestrado, DEMa/UFSCar , maio (1997).
16. Mo, Z. e Zhang, H., J. Macromol. Sci. - Rev. Macromol. Chem. Phys., C35(4), p. 555-580 (1995).

17. Rabiej, S. Eur. Polym. J., 27, n.9, p. 947-954 (1991).

18. Bain, L. J. "Statistical Analysis of Reliability and Life-Testing Models", New York: Marcel Dekker, Inc. vol. 24, 450 p, (1978).

19. Ueki, M. M. e Zanin, M., Anais dol $12^{\circ}$ CBECIMAT, Águas de Lindóia - SP, dezembro (1996) p. 1346-1349.

20. Ogbobe, O., J. Appl. Polym. Sci., 49, p. 381-389 (1993).

21. Tadmor, Z. e Gogos, C. G., "Principles of Polymer Processing”, USA: John Wiley \& Sons, 735 p, (1979).

22. Rwei, S. P., Manas-Zloczower, I. e Feke, D. L., Polym. Eng. Sci., 31, p. 558-562 (1991). 